\title{
CARVEDILOL NA HIPERTROFIA CARDÍACA INDUZIDA POR ANABOLIZANTES EM COELHOS
}

\section{Carvedilol in cardiac hypertrophy due to use of anabolisants in rabbits}

\author{
MEIRELLES, A.C.F'; ZANOTTO, G. ${ }^{2}$; WOUK, A.F.P.F ${ }^{2}$ \\ ${ }^{1}$ Pontifícia Universidade Católica do Paraná - Campus Toledo \\ ${ }^{2}$ Departamento de Medicina Veterinária - Universidade Federal do Paraná \\ Endereço para correspondência: Andrea Meirelles - acmeirelles@hotmail.com
}

\section{RESUMO}

Estudou-se a ação do carvedilol frente a alterações cardiovasculares induzidas pelo uso de esteróides anabólicos androgênicos (EAA), em 30 coelhos. O monitoramento cardíaco deu-se fundamentalmente pela análise da silhueta cardíaca a partir de radiografias seqüenciais mensais, analisadas através do programa AUTOCAD $2004^{\circledR}$. O uso do carvedilol reduziu em 13\% em média a hipertrofia miocárdica secundária ao uso de $5 \mathrm{mg}$ de decanoato de nandrolona, semanalmente, durante nove semanas, proporcionando remodelamento da silhueta cardíaca, com diminuição das áreas, perímetros e volumes cardíacos, quando analisadas com o auxílio do AUTOCAD 2004 ${ }^{\circledR}$. A interpretação do remodelamento cardíaco com o AUTOCAD $2004^{\circledR}$ pode ser adotada como prática diagnóstica para a investigação de pacientes com cardiomiopatias pela sua versatilidade, baixo custo e por proporcionar parâmetros fidedignos de evolução de cardiopatias. $\mathrm{O}$ carvedilol é uma opção a ser considerada para o tratamento das cardiomiopatias hipertróficas.

Palavras-chave: $\beta$-bloqueador, decanoato de nandrolona, remodelamento miocárdico.

\begin{abstract}
With the intention of testing the use of carvedilol for the treatment of secondary cardiovascular alterations due to the use of AAS, an experimental study was accomplished in 30 rabbits. Heart monitoring was performed through the analysis of heart silhouette obtained from a sequence of $x$-rays and evaluated through the program AUTOCAD $2004^{\circledR}$. The use of carvedilol reduced in $13 \%$ in average the secondary myocardial hypertrophy to the use of $5 \mathrm{mg}$ of nandrolone decanoate, weekly, during 9 weeks, providing remodelamento of the cardiac silhouette, with reduction of the areas, cardiac perimeters and volumes, when analyzed with the aid of the AUTOCAD 2004®. The use of carvedilol decrease in $13 \%$ the hypertrophy secondary to the chronic use of nandrolone decanoate in the dosage employed of $5 \mathrm{mg}$ for 9 weeks, remodeling of the cardiac silhouette. A decrease of areas, perimeters and heart size was observed when analyzed with the AUTOCAD $2004^{\circledR}$ program. The interpretation of heart remodulation with the use of AUTOCAD $2004^{\circledR}$ could be adopted in the veterinary medicine practice for the evaluation of the treatment of patients with long period cardiomyopathy, because its great versatility, low costs and for providing trustworthy parameters of evolution of the hipertrofic cardiomiopathy.
\end{abstract}

Key words: $\beta$-blocker; nadrolone decanoate; miocardic remodulation. 


\section{INTRODUÇÃO}

O primeiro uso não-médico dos esteróides anabólico-androgênicos (EAA) foi feito por soldados alemães na II Guerra Mundial com 0 intuito de aumentar a agressividade (Fuller, 1993). O ano de 1975 ficou marcado pela inclusão dos EAA na lista de drogas consideradas doping pelo Comitê Olímpico Internacional, sendo o ano de 1988 um marco histórico desta questão, pois foi quando o atleta Ben Johnson perdeu sua medalha olímpica em Seul, devido ao uso de EAA (Fuller, 1993; Kennedy, 2000). Um estudo populacional realizado em 1988 nos EUA estimou em mais de um milhão os usuários de EAA. Mesmo não havendo dados epidemiológicos brasileiros, esses estudos nos dão idéia da dimensão do problema.

O aumento do consumo de anabolizantes em grupos que não buscam a melhora do rendimento, mas se preocupam com a imagem do corpo ideal, tornou-se um problema de saúde pública mundial (American College of Sports Medicine, 1987; Porcerelli e Sandler, 1998).

Desde que a combinação de $\beta$ bloqueadores e vasodilatadores têm demonstrado ser efetiva em diminuir a pressão sangüínea e manter 0 débito cardíaco, novos $\beta$-bloqueadores com adicional propriedade de vasodilatação, como o carvedilol, são intensamente pesquisados (Höher et al., 1989).

O carvedilol atua favoravelmente reduzindo o estresse oxidativo e injúria mitocondrial. Em cães com cardiomiopatia dilatada, este proporciona aumento da força de contração, melhora 0 débito cardíaco, remodelamento miocárdico e diminuição da área e perímetro cardíaco, proporcionando aos pacientes melhora na qualidade de vida (Wendt, 2003).
É provável que os $\beta$-bloqueadores tenham, em coelhos, os mesmos efeitos que já demonstraram em seres humanos e cães. No entanto, não existem estudos substanciais avaliando 0 efeito remodelador deste fármaco nesta espécie. A fim de avaliar a capacidade do carvedilol no remodelamento miocárdico e diminuição da silhueta cardíaca induzida experimentalmente pelo uso crônico de anabolizante, foi utilizado um modelo experimental lagomorfo com coelhos (Oryctolagus cuniculus) submetidos a altas doses de decanoato de nandrolona, comparativamente ao que ocorre em seres humanos.

\section{MATERIAL E MÉTODOS}

Foram utilizados 30 coelhos (Oryctolagus cuniculus) machos, adultos, Nova Zelândia, brancos, albinos, com idade aproximada de 120 dias, divididos inicialmente em dois grupos: Grupo Nandrolona com 20 animais (ANA) e Grupo Placebo com 10 animais (PLA), provenientes de um biotério particular e alojados nas dependências do Hospital Veterinário da UFPR.

\section{Fase 1 - Anabolizante}

Inicialmente para indução da hipertrofia cardíaca, o grupo ANA (20 animais) recebeu injeções intramusculares, no músculo quadríceps femoral, de decanoato de nandrolona na dose de 5 $\mathrm{mg} / \mathrm{kg}$ uma vez por semana, dose obtida por extrapolação alométrica, durante nove semanas de tratamento, conforme protocolo terapêutico descrito por Trifunovic et al. (1995). O grupo PLA (10 animais) recebeu uma vez por semana, durante nove semanas consecutivas injeções intramusculares de óleo vegetal na mesma quantidade do fármaco (Damasceno, 2003). Optou-se por utilizar este veículo, 
visto a droga a ser administrada, ser uma solução igualmente oleosa. Desta maneira o estresse desencadeado pelas injeções seria comparável.

\section{Fase 2 - Carvedilol}

Depois da fase anabolizante os 20 animais que receberam decanoato de nandrolona foram divididos em 2 grupos iguais - grupos CAR (carvedilol) e CON (controle). Os animais do Grupo PLA da Fase I foram doados para o biotério de origem. Procedeu-se então o tratamento com o carvedilol ao grupo CAR na dose de dois $\mathrm{mg} / \mathrm{kg}$ diariamente, por via oral, suspensão manipulada a $25 \mathrm{mg} / \mathrm{ml}$, durante 90 dias. Os animais do grupo CON não receberam tratamento.

\section{Avaliações}

Os animais foram radiografados em seis momentos diferentes, antes de iniciarem os protocolos e depois durante todo o período experimental a cada 30 dias.

- Ao início do tratamento Anabolizante ou Placebo - M1 -ANA

- 30 dias após o início dos tratamentos M2-ANA

- Final do tratamento Anabolizante $\mathrm{x}$ Início do Tratamento Carvedilol - M3ANA

- 30 dias após o início do tratamento Carvedilol - M4-CAR

- 60 dias após o início do tratamento Carvedilol - M5-CAR

- 90 dias após o início do tratamento Carvedilol - M6-CAR
Avaliação Radiográfica - O exame radiográfico torácico foi realizado no Serviço de Radiologia do Hospital Veterinário da UFPR, nas exposições dorso-ventral e látero-lateral de segundo Lord e Suter (1999). As radiografias foram avaliadas pelo método comparativo de análise de imagem e analizadas com 0 programa computadorizado AUTOCAD $2004^{\circledR}$ que possibilita obtenção da área, perímetro e volume cardíaco de imagens radiográficas prévias do mesmo animal (Wendt, 2003).

Conversão Analógico-digital das Imagens Radiografias - Para a obtenção das áreas cardíacas foi necessário converter a imagem do formato analógico com uma câmera digital. Para tanto, a radiografia foi afixada sobre uma bancada e posicionada sob um suporte de fixação para a câmera. O suporte tem por objetivo fixar uma distância para o objeto e desta forma padronizar a tomada das informações (Figura 1).

Interpretação da Imagem e Obtenção da Informação Vetorial - Como a imagem digital está no formato matricial, era necessário converter a informação para o formato vetorial $(x, y, z)$. Para tanto foram empregadas ferramentas para criação de vetores, disponível no programa AUTOCAD ${ }^{\circledR}$. Adicionalmente, foram empregadas as ferramentas de "zoom" visando delinear corretamente o coração. Cada coração delineado resultou em um polígono bidimensional.

Obtenção das Áreas, Perímetros e Volumes delineada - Concluída a vetorização das imagens, procedeu-se à extração das informações referentes às áreas, perímetros e volumes de cada coração. Foi utilizada linguagem de programação para extração automática a partir dos vetores de cada imagem (Figura 2). 


\section{Histopatologia}

A histopatologia foi utilizada para definir o tipo de alteração miocárdica decorrente do uso do decanoato de nandrolona. Os animais dos grupos CAR e CON foram submetidos à eutanásia, com injeção intravenosa letal de pentobarbital sódico. Os corações foram retirados, acondicionados em frascos plásticos individuais, devidamente identificados e contendo solução neutra de formol $10 \%$, para realização da técnica histopatológica (Bacha e Wood, 1990).

Nos corações dos coelhos do grupo CON não foram observadas alterações dignas de nota.

Nos corações do grupo CAR observou-se fibras musculares cardíacas dispostas irregularmente, aumentadas de volume, núcleo hipercromático. Notou-se que o miocárdio encontrava-se constituído, principalmente, de fibras musculares cardíacas, capilares e substância intercelular. Com relação à orientação dos cardiomiócitos na parede do ventrículo esquerdo, verificou-se que estes se encontram agrupados, formando feixes em várias direções do espaço, ou seja, em determinados campos do microscópio observam-se feixes cortados transversalmente ao lado de outros dispostos longitudinalmente ou mesmo obliquamente, obtendo-se o Laudo Histopatológico de Hipertrofia Miocárdica.

\section{Avaliação estatística}

Utilizou-se um delineamento
inteiramente ao acaso com parcelas
subdivididas no tempo, sendo a da Fase 1
(anabolizante) e a Fase 2 (carvedilol)
compostas por três repetições cada. Os
resultados foram submetidos à análise de
variância dos tratamentos quanto a sua
homogeneidade pelo teste de variância
ANOVA: Fatorial (a x b) com replicação.
Quando os resultados revelaram existir

diferenças estatisticamente significativas entre médias de tratamentos, as médias foram comparadas pelo teste de Tukey no nível de $5 \%$ de probabilidade $(p<0,05)$. Para a realização da análise estatística foi utilizado o programa computadorizado BioEstat versão 3.0 (AYRES et al., 2003).

\section{RESULTADOS E DISCUSSÃO}

\section{Análise das Imagens Radiográficas}

\section{ÁREAS}

Em M2 x M3 e M5 x M6, as médias demonstram diferenças estatisticamente significativas dos perímetros $(\mathrm{P}<0,05)$. Em M1, M2, M3 observou-se aumento das áreas, o que corresponde ao período de

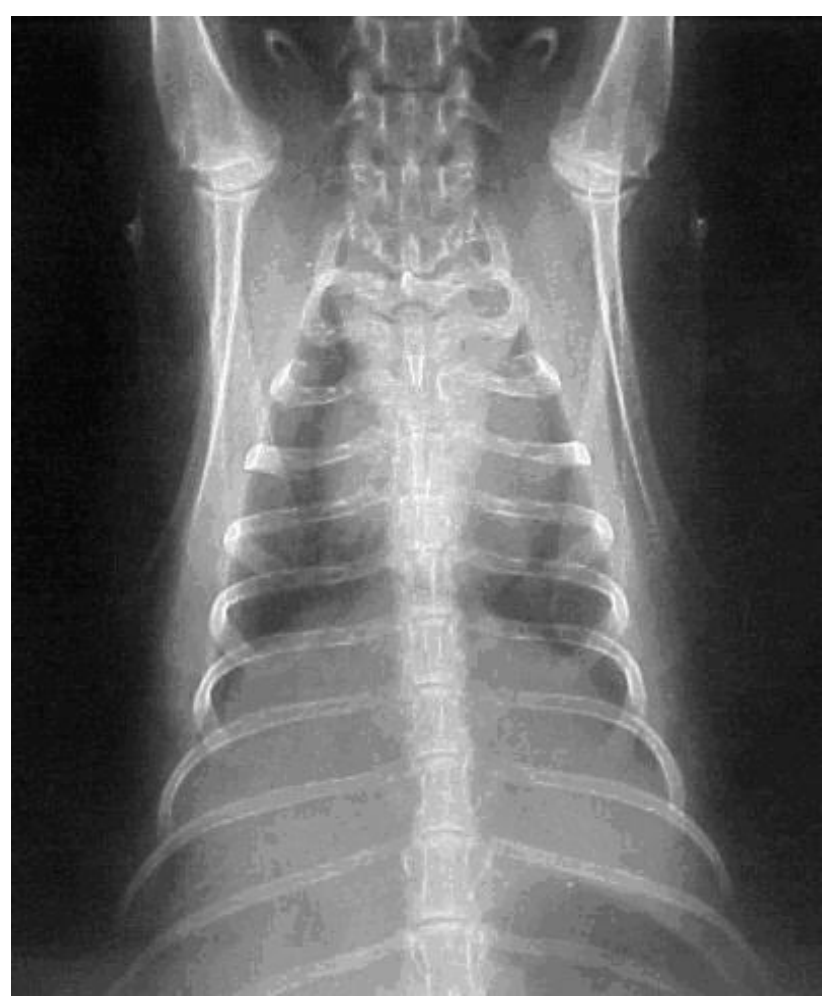

Figura 1 - Aspecto radiográfico da silhueta cardíaca, em incidência dorso-ventral de um coelho macho. 
uso do Anabolizante. Pelos valores expressos das medidas encontradas temos evidências de diminuição significativa das médias das áreas cardíacas, tendo-se uma tendência de diminuição destes valores durante o período de uso do carvedilol. No figura 3 verifica-se a variação dos valores médios da áreas cardíacas em $\mathrm{mm}^{2}$ dos animais estudados durante todo o período experimental (média \pm desvio padrão).
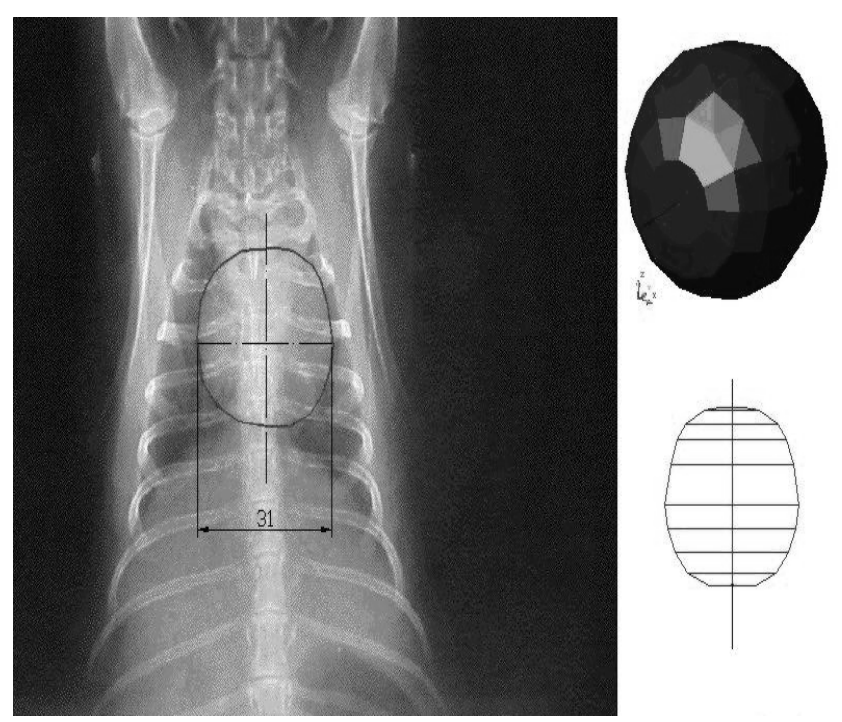

Figura 2 - Aspecto radiográfico da silhueta cardíaca, incidência dorso-ventral, de um coelho, macho, analisada pelo AUTOCAD $^{\circledR}$ com perímetro $117,43 \mathrm{~mm}$, área $1075,93 \mathrm{~mm}^{2}$, e volume 23722,28 $\mathrm{mm}^{3}$.

\section{PERÍMETROS}

Em M1 x M2 e M5 x M6, as médias demonstram diferenças estatisticamente significativas dos perímetros $(p<0,05)$. Momentos que correspondem ao início do uso do Anabolizante e ao período final de uso do carvedilol. Na figura 4 verifica-se a variação dos valores médios dos perímetros cardíacos em $\mathrm{mm}$ dos animais estudados durante todo 0 período experimental (média \pm desvio padrão).

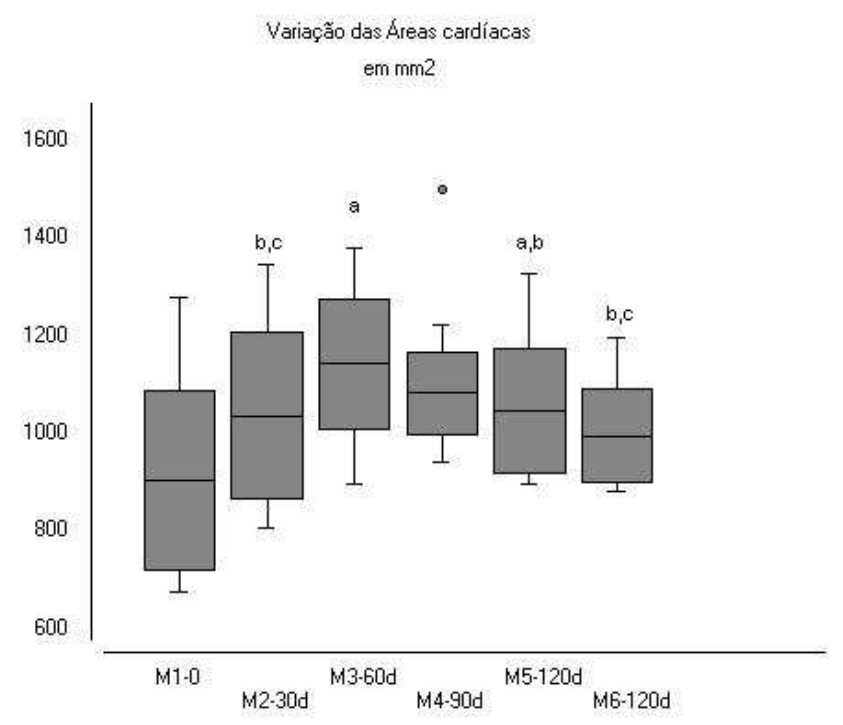

Notas: ${ }^{a, b, c}$ - Médias seguidas de letras minúsculas diferentes no gráfico diferem entre si a $5 \%$ de significância segundo teste Tukey. Resultados referentes aos animais que receberam Decanoato de nandrolona e posteriormente tratamento com Carvedilol até o final do período experimental.

Figura 3 - Variação dos valores médios das áreas cardíacas dos animais estudados durante todo o período experimental (0,30,60, 90, 120 e 15 dias).

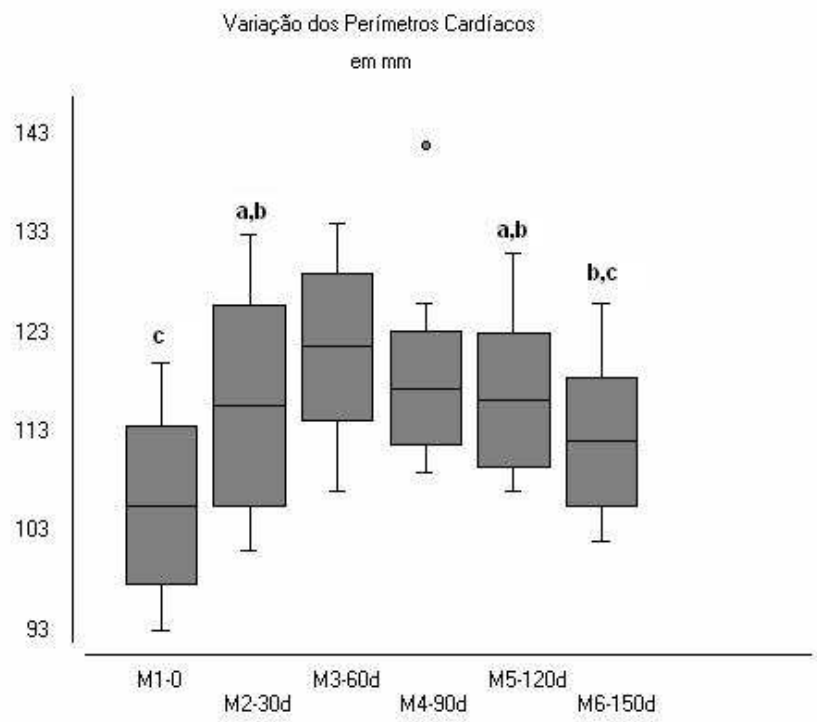

Notas: ${ }^{\text {a, b, c }}$ - Médias seguidas de letras minúsculas diferentes no gráfico diferem entre si a $5 \%$ de significância segundo teste Tukey. Resultados referentes aos animais que receberam decanoato de nandrolona e posteriormente tratamento com carvedilol até o final do período experimental.

Figura 4 - Variação dos valores médios dos perímetros cardíacos dos animais estudados durante todo o período experimental $(0,30,60,90$, 120 e 15 dias). 


\section{VOLUMES}

Foi encontrada diferença estatisticamente significativa na análise dos volumes dos grupos ANA e CAR quando comparados os momentos M1 x M2; M2 x M3; M5 x M6 $(p<0,05)$. Pelos resultados encontrados observa-se que houve um aumento de volume cardíaco gradual com o uso de anabolizante e uma discreta diminuição destes valores com a utilização do carvedilol. Nas TABELAS 1 e 2 verificase a variação dos valores médios do volumes cardíacos em $\mathrm{mm}^{3}$ dos animais estudados durante todo 0 período experimental (média \pm desvio padrão).

Tabela 1 - Volume dorso-ventral (VOL) em $\mathrm{mm}^{3}$, no início e no final do tratamento anabolizante (GRUPO ANA), obtendo-se o percentual de remodelamento, após análise das radiografias pelo programa AUTOCAD $2004^{\circledR}$.

\begin{tabular}{cccc}
\hline Animais & VOL inicial & VOL final & Remodelamento \\
\hline C01 & 21680,51 & 24042,25 & $7,18 \%$ \\
C02 & 14432,63 & 26829,44 & $72,56 \%$ \\
C03 & 13653,67 & 23544,15 & $71,1 \%$ \\
C04 & 19825,09 & 25589,15 & $21,1 \%$ \\
C05 & 19825,09 & 25346,01 & $19,7 \%$ \\
C06 & 16235,85 & 26242,11 & $27,5 \%$ \\
C07 & 12377,95 & 18253,2 & $67 \%$ \\
C08 & 14768,94 & 23303,44 & $32,8 \%$ \\
C09 & 20316,77 & 33601,01 & $74,3 \%$ \\
C10 & 24362,18 & 29063,80 & $9,6 \%$ \\
Média & $\mathbf{1 7 7 4 7 , 8 7 ^ { b }}$ & $\mathbf{2 5 5 8 1 , 4 6}$ & $\mathbf{3 0} \%$ \\
Desvio Padrão & 3974,82 & 3998,63 & $27 \%$ \\
\hline
\end{tabular}

Notas: VOL: Volume, C: Coelho; ${ }^{\mathrm{a}, \mathrm{b}}$ - Médias seguidas de letras minúsculas diferentes na linha diferem entre si a 5 \% de significância segundo teste Tukey.

Tabela 2 - Volume dorso-ventral (VOL) em $\mathrm{mm}^{3}$, no início e final do tratamento carvedilol (CAR), obtendo-se o percentual de remodelamento, após análise das radiografias pelo programa AUTOCAD $2004^{\circledR}$.

\begin{tabular}{cccc}
\hline Animais & VOL inicial & VOL final & Remodelamento \\
\hline C01 & 23237,04 & 20029,62 & $16,01 \%$ \\
C02 & 24904,68 & 18375,03 & $35,53 \%$ \\
C03 & 23358,51 & 14475,5 & $61,36 \%$ \\
C04 & 24004,37 & 23722,28 & $1,18 \%$ \\
C05 & 23722,28 & 13598,20 & $74,45 \%$ \\
C06 & 20698,97 & 20356,79 & $16,53 \%$ \\
C07 & 20667,59 & 17572,13 & $17,61 \%$ \\
C08 & 19613,43 & 19990,77 & $1,92 \%$ \\
C09 & 35402,29 & 28444,94 & $24,45 \%$ \\
C10 & 26691,65 & 25226,28 & $5,80 \%$ \\
Média & $\mathbf{2 4 2 3 0 , 0 8 ^ { \mathbf { a } }}$ & $\mathbf{2 0 1 7 9 , 1 5 ^ { \mathbf { b } }}$ & $\mathbf{1 3 , 7 8} \%$ \\
Desvio Padrão & 4470,40 & 4622,79 & $24,84 \%$
\end{tabular}

Notas: VOL: Volume, C: Coelho; ${ }^{a, b}$ - Médias seguidas de letras minúsculas diferentes na linha diferem entre si a $5 \%$ de significância segundo teste Tukey. 


\section{DISCUSSÃO}

Este estudo foi aprovado pelo Comitê de Ética em Pesquisas com Animais da PUC-PR (CEPA).

No presente trabalho, estudou-se a ação do carvedilol em alterações cardiovasculares induzidas pelo decanoato de nandrolona em coelhos. A revisão da literatura não evidenciou publicações com os objetivos e metodologias superdisponíveis a este estudo, o que sugere esta ser uma abordagem original.

A dose utilizada do decanoato de nandrolona foi de cinco $\mathrm{mg} / \mathrm{kg}$ de peso semanalmente e conforme a literatura consultada, essa dose é considerada elevada (Trifunovic et al., 1995). Esta dose foi baseada em prática rotineira de atletas e não atletas interessados em ter um desempenho físico melhorado (Guimarães Neto, 2000; Llewellyn, 2000), e obtida através de extrapolação alométrica segundo Pachaly (2000).

Os animais não foram treinados a realizar qualquer tipo de atividade física com o objetivo de não se criar um viés no estudo da hipertrofia cardíaca, visto a prática esportiva levar ao aparecimento de alterações funcionais e anatômicas neste órgão, aumentando a sensibilidade dos receptores androgênicos, já que são muitos os mecanismos adaptativos do coração em resposta ao crescimento, ao exercício e outras mudanças fisiológicas que estão sob influência dos esteróides, sejam eles endógenos ou não (Kleiner et al., 1989; Rockhold, 1993; Melchert et al., 1995; Sullivan et al., 1998).

\footnotetext{
Corroborando com os resultados deste estudo, diversos modelos experimentais realizados em várias espécies animais demonstram claramente - aparecimento de cardiomegalia após algumas semanas de uso de EAA (Pesola, 1988; De Picolli et al., 1991).
}

Os resultados dos exames histopatológicos revelaram hipertrofia miocárdica com fibras musculares cardíacas dispostas irregularmente e aumentadas de volume, estes achados corroboram com Damasceno (2003) que em um estudo similar com ratos sensibilizados com altas doses de decanoato de nandrolona observou hipertrofia miocárdica ventricular esquerda com auxílio da análise de seus miocárdios por video-histomorfometria.

Até este momento, não existe consenso científico sobre a persistência destas alterações após a descontinuidade da droga e de seus efeitos em longo prazo, devido aos poucos estudos de acompanhamento de uso prolongado dos EAA. Porém, Urhausen et al. (2004) e Payne (2004) demostraram em um estudo recente que os efeitos adversos do uso de esteróides anabolizantes sobre a estrutura cardíaca podem ser vistos durante vários anos depois da interrupção do uso dos agentes.

O carvedilol foi utilizado neste trabalho com o objetivo de estabilizar e reduzir 0 remodelamento ventricular desencadeado pelo uso do EAA. Este fármaco foi utilizado com sucesso na estabilização e redução da silhueta cardíaca de cães com cardiomiopatia dilatada em um estudo clínico realizado por Wendt (2003).

Embora vários pesquisadores focados em diferentes propostas tenham tentado comprovar o exato mecanismo de atuação do carvedilol para reverter ou prevenir o remodelamento miocárdico adverso e reduzir a hipertrofia miocítica, este mecanismo parece ser multifatorial (Wendt, 2003; Wang et al., 2004).

Uma hipótese para a ação do carvedilol é a da redução da sobrecarga ventricular esquerda, associada à inibição dos fatores estimulantes de crescimento e 
injúria dos cardiomiócitos e fibroblastos devido a sua potente atividade antioxidante (Wang et al., 2004).

O método de análise morfométrica radiográfica com 0 auxílio do AUTOCAD $2002^{\circledR}$ proposto por Wendt (2003) foi adaptado para este estudo com uma versão mais moderna que possibilitou além de mensurações de áreas e perímetros também obter 0 volume dos corações radiografados.

Houve redução significativa das áreas, perímetros e volumes cardíacos ao final do tratamento com carvedilol, isto vai de encontro aos achados de Wendt (2003), que em um estudo clínico similar com cães com cardiomiopatia dilatada obteve remodelamento e redução da silhueta cardíaca em incidência látero-lateral e dorso-ventral.

O tempo de seguimento e o número de pacientes não são suficientes para conclusão definitiva a respeito de efeitos na mortalidade em longo prazo. Entretanto, os efeitos obtidos na melhora clínica sugerem um efeito benéfico já que esta é importante na determinação do prognóstico.

\section{CONCLUSÕES}

O uso do Carvedilol em coelhos com hipertrofia miocárdica secundária ao uso crônico de esteróides anabólicos androgênicos reduz a hipertrofia cardíaca em $20 \%$ em média e por isto é uma opção de tratamento para as cardiomiopatias dilatadas androgênicas. A análise morfométrica de imagens radiográficas pelo AUTOCAD $2004^{\circledR}$ é mais uma ferramenta diagnóstica, pela sua versatilidade, baixa custo e eficácia, pois proporciona parâmetros morfométricos confiáveis do coração.

\section{CONSIDERAÇÕES PROSPECTIVAS}

Em estudo futuros com base nos resultados aqui obtidos, poderão ser incorporados à metodologia os seguintes métodos de avaliação para validar a técnica de Análise Morfométrica com auxílio do AUTOCAD $2004^{\circledR}$ :

- Morfometria cardíaca direta;

- Ecocardiografia com doppler colorido;

- Tomografia Computadorizada;

- Vídeo-histomorfometria cardíaca;

\section{REFERÊNCIAS}

AMERICAN COLLEGE OF SPORTS MEDICINE. Position statement on the use and abuse of anabolic-androgenic steroids in sports. Medicine \& Science in Sports \& Exercise, n. 19, p.534-539, 1987. Disponível em: <http//www.acsm.org/> Acesso em: 01 ago 2005.

AYRES, M.; AYRES, D. L.; SANTOS, A.S. Aplicações estatísticas nas áreas das ciências biológicas e médicas. Bio Estat 3.0, Belém: Sociedade Civil Mamirauá, 2003.

BACHA, J. R.; WOOD, L. M. Color Atlas of
Veterinary Histology. Philadelphia: Williams \& Wilkins, 1990.

BROWER, K. J.; BLOW, F. C.; BERESFORD, T. P.; FUELLING, C. Anabolic-androgenic steroid dependence. Journal of Clinical Psychiatry, vol. 1, n. 50, p. 31-33, 1989.

COUNCIL ON SCIENTIFIC AFFAIRS. Medical and nonmedical uses of anabolicandrogenic steroids. Journal of the 
American Medical Association, v. 264, n.22, p. 2923-2927, 1990.

DAMASCENO, M.C.T. Prevenção do remodelamento ventricular esquerdo induzido pelo decanoato de nandrolona. São Paulo, 2003. 75 f. Dissertação (Mestrado em Emergências Clínicas) Faculdade de Medicina da Universidade de São Paulo.

DE PICOLI, B.F.; GIADA, F.; BENETETTIN, A. Anabolic steroid use in body builders: an echocardiographic study of left ventricular morphology and function. International Journal of Sports Medicine, v. 12, n. 4, p. 408-412, 1991.

FERENCHICK, G.S. Association of steroid abuse with cardiomyopathy in athletes. American Journal of Cardiology, v.91, n. 5, p. 588-596, 1991.

FULLER, M.G.; Anabolic-androgenic steroids: use and abuse. Comprehensive Therapy, v. 19, n. 2, p. 69-72, 1993.

GUIMARÃES NETO, W.M. Musculação Anabolismo Total. 4. ed. São Paulo: Editora Phorte, 2000.

HÖHER, M.; FRIEDRICH, M.; SOMMER, T.; MARTEN, A.; EHMER, B.; HOMBACH, V.; HIRCHE, HJ. Effects of carvedilol on left ventricular function and arrhythmias during repeated short-time myocardial ischemia in experimental pigs. Kardiologie, n. 78: Suppl. 3, p. 7-15, 1989.

JOHNSON, W.O. Steroids: a problem of huge dimensions. Sports Illustrated, v.5, n.13, p.38-54, 1985.

KENNEDY, M. C. Newer drugs used to enhance sporting performance. The Medical Journal of Australia, v.173, n. 6, p. 314-317, 2000.

KLEINER, S.M.; CALABRESE, L.H.; FIELDER, K.M. Dietary influences on cardiovascular disease risk in anabolic steroid-using and no using bodybuilders. Journal of the American College Nutrition, v. 08, n. 2, p. 109-119, 1989.

LLEWELLYN, W. Anabolic Steroid Reference Manual - Anabolics 2000, 1. ed. Estados Unidos, 2000.

LORD, P. F.; SUTER, P. F. Radiology. In:

FOX, P. R.; SISSON, D.; MOISE, S. N.

Textbook of Canine and Feline

Cardiology - Principles and Clinical

Practice. Philadelphia: W. B. Saunders

Company, cap. 7, p. $107-129,1999$.

LUKE, L.J.; FARB, A.; VIRMANI, R.; SAMPLE, R.H.B. Sudden cardiac death during exercise in a weight lifter using anabolic androgenic steroids: pathologic and toxicological findings. Journal -

Forensic Science Society, v. 35, p. 14411447, 1990.

MEHVAR, R.; BROCKS, D.R. Stereospecific Pharmacokinetics and Pharmacodynamics of Beta-Adrenergic Blocker in Humans. Journal Pharmacology Pharmaceutical Science, v. 4, n. 2, p. 185-200, 2001. Disponível em: $<$ http://www.ualberta.ca/ csps/JPPS4(2)/R.Meh var/betablockers.htm> Acesso em: 01 ago. 2005.

MELCHERT, R.B.; WELDER, A.A. Cardiovascular effects of androgenic anabolic steroids. Medicine Science of Sports and Exercise, v.27, n. 9, p. 12521262, 1995.

PACHALY, J. R. Emprego do método de extrapolação alométrica no cálculo de protocolos posológicos para animais selvagens. A Hora Veterinária, v. $20, \mathrm{n}$. 118, p. 59-65, 2000.

PAYNE, J. R.; KOTWINSKI, P. J.; MONTGOMERY, H.E. Cardiac effects of anabolic steroids, Heart, v. 90, London, p. 473-475, 2004. 
PESOLA, M.K. Reversibility of the haemodynamic effects of anabolic steroids in

rats.

European Journal of Applied Physiology, v. 58, n. 1, p. 125-131, 1988.

POPE, H. G.; PHILLIPS, K. A.; OLIVARDIA, R. O. O Complexo de Adonis - a obsessão masculina pelo corpo, 1. ed. Rio de Janeiro: Editora Campus, 2000.

PORCERELLI, J.H.; SANDLER, B.A. Anabolic-androgenic steroid abuse and psychopathology. The Psychiatry Clinics of North America, v. 21, n. 4, p.829-833, 1998.

ROCKHOLD, R.W. Cardiovascular toxicity of anabolic steroids. Annual Review of Pharmacology and Toxicology, v. 33, p. 497-520, 1993.

SILVA, P.R.P.; DANIELSKI, R.; CZEPIELEWSKI, M.A. Esteróides anabolizantes no esporte. Revista Brasileira de Medicina no Esporte, v. 8, n. 6, p. 235-243, 2002. Disponível em: $<$ http:// www.revista brasileira de medicina do esporte - esteróides anabolizantes no esporte-b.htm>Acesso em: 9 set. 2005.

SULLIVAN, M.L.; MATINEZ, C.M.; GENIS, P.; GALLAGHER, E.J. The Cardiac Toxicology of Anabolic Steroids. Progress in Cardiovascular Diseases, v. 41, p. 1 15, 1998.

THOMPSON, P.D.; SADANIANTZ, A.; CULLINANE, E.M.; BODZIONY, K.S.; CATLIN, D.H.; TOREK-BOTH, G.; DOUGLAS, P.S. Left ventricular function is not impaired in weight-lifters who use anabolic steroids. Journal of American College Cardiology, v. 19, n. 2, p. 278282, 1992.

TRIFUNOVIC, B; NORTON, G.R.;

DUFFIELD, M.J. An androgenic steroid decreases left ventricular compliance in rats. Journal of Applied Physiology, v. 01, n. 3, p. 1096-1105, 1995.

URHAUSEN, A.; ALBERS, T.; KINDERMANN, W. Are the cardiac effects of anabolic steroid abuse in strength athletes reversible? Heart, v. 90, n. 5, p. 496-501, 2004.

WANG, J. K.; CUI, C. C.; ZHANG, H.; YAO, Q. H.; YAO, X.W.; CHEN, X. Y. Adrenergic receptor antagonist prevents the left ventricle with chronic pressure-overload from electrical remodeling. Acta Physiologica Sinica, v. 56, n. 4, p.487492, 2004.

WENDT, H. Estudo do uso do carvedilol em cães com cardiomiopatia dilatada. Curitiba, 2003. 144p. Dissertação (Mestrado em Ciências Veterinárias) - Setor de Ciências Agrárias, Universidade Federal do Paraná. 